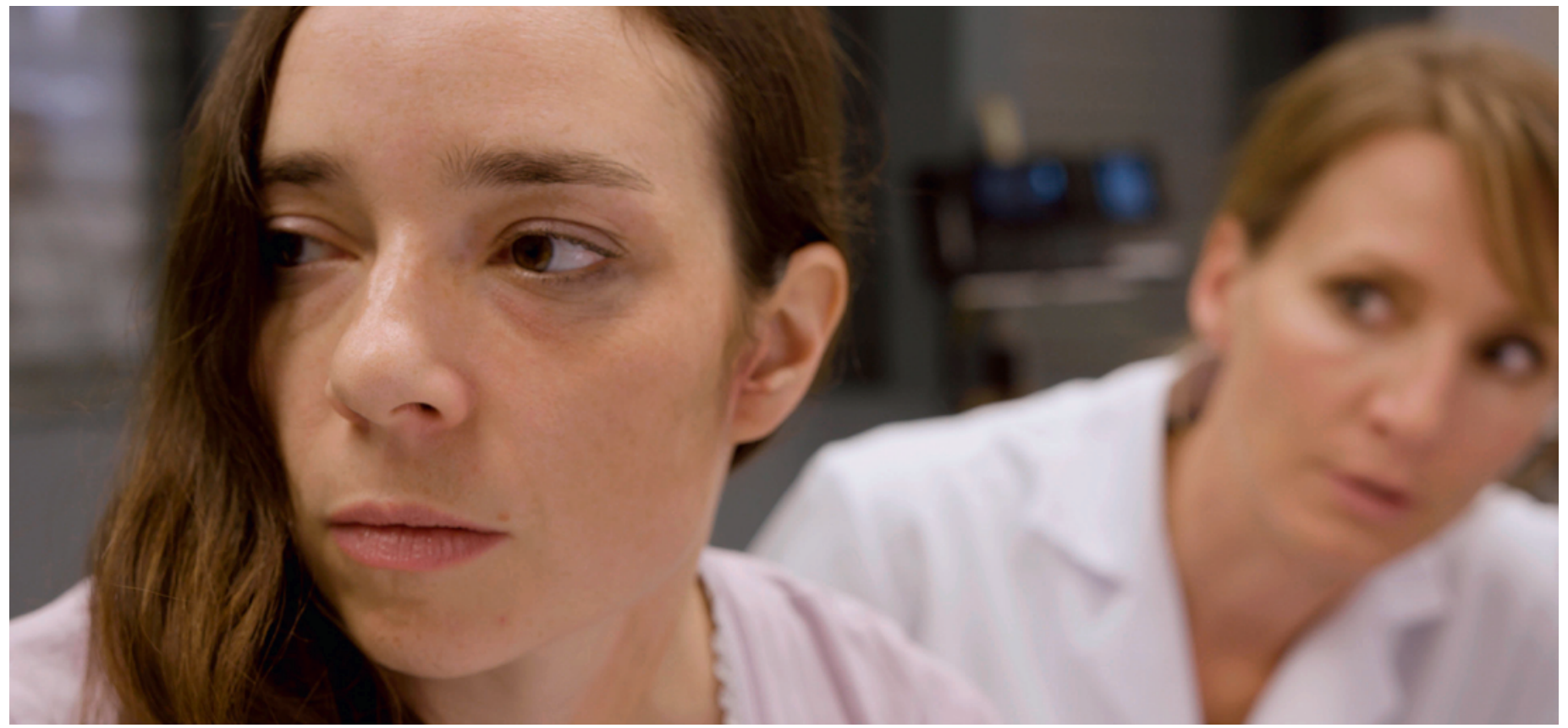

\title{
Und wenn diese Patientin ein Menschenhandelsopfer ist?
}

\section{Anne-Florence Débois}

Mediensprecherin, Bundesamt für Polizei (fedpol)

Menschenhandel ist eine Realität in der Schweiz. 2017 wurden 149 Menschen Opfer von sexueller Ausbeutung oder von Ausbeutung der Arbeitskraft. Wenn ihnen körperliche Gewalt angetan worden ist, werden sie manchmal von ihrem Peiniger zur Behandlung ins Spital begleitet. Sie als medizinische Fachkraft spielen bei der Untersuchung eine entscheidende Rolle. Damit Sie Opfer von Menschenhandel erkennen und ihnen helfen können, startet fedpol eine Sensibilisierungskampagne.

In ihrem Gesicht sind keine Anzeichen sichtbar, die auf körperliche Gewalt hindeuten, wohl aber zahlreiche blaue Blutergüsse am Rücken. Auch auf dem Bauch der Patientin sind Prellungen zu erkennen; sie haben mittlerweile eine gelbliche Farbe angenommen. Die Urinanalyse zeigt, dass die Frau an einer schwerwiegenden Harnwegsinfektion leidet. Auf den ersten Blick lässt sich schwer sagen, welche Umstände sie dazu gebracht haben, ins Spital zu kommen. Sie spricht kein Deutsch. Ihr männlicher Begleiter - er gibt sich als ein Freund aus - besteht darauf, bei der Untersuchung dabei zu sein.
Tatsache ist: Diese Frau ist ein Opfer von Menschenhandel. Sie wird sexuell ausgebeutet; vor einigen Tagen ist sie geschlagen worden. Kein Abend, ohne dass ihr nicht gedroht wird, falls sie nicht auf den Strassen-

Diese Frau ist ein Opfer von Menschenhandel.

Sie wird sexuell ausgebeutet.

strich geht. Dieses Mal ist ihr Zuhälter gewalttätig geworden. Er hat sie auf den Rücken geschlagen, weil sie nicht genügend Geld angeschafft hat. Ganz abgesehen vom Trauma, das sie erlitten hat, weil sie mehrere Mo- 
nate zuvor ihre Familie und Eltern verlassen musste, in der Hoffnung, in der Schweiz eine richtige Arbeit zu finden und eine neue Existenz aufzubauen - ein Leben ohne Not.

Als Ärztin oder Arzt, Pflegefachkraft oder Praxisassistentin oder -assistent: Hätten Sie bemerkt, dass die Patientin ein Menschenhandelsopfer ist? Und wie könnten Sie diesem Menschen dabei helfen, sich aus den Fängen seines Peinigers zu befreien? Was können Sie ganz konkret für ihn tun?

\section{Sie spielen eine entscheidende Rolle}

Ende Juni startete fedpol im Berner Inselspital eine nationale Sensibilisierungskampagne. Diese Kampagne richtet sich an medizinisches und pflegerisches Fachpersonal und soll auf ein Thema aufmerksam machen, das noch immer ein Tabu ist, aber nicht ignoriert werden darf. Auch die Schweiz ist von Menschenhandel betroffen. Es ist eine Tatsache: Die Ausbeuter begleiten ihre Opfer, die verletzt oder bei schlechter Gesundheit sind, ins Spital. Schliesslich ist es in ihrem eigenen Interesse, dass sie medizinisch behandelt werden. Die Opfer werden vorzugsweise in grosse Spitäler oder medizinische Notfallzentren gebracht, denn hier besteht weniger Gefahr aufzufallen. Die Untersuchung im Spital oder in der Notaufnahme bietet eine erste Gelegenheit, um Menschenhandelsopfer zu identifizieren. Vor allem aber bietet sich den Opfern eine Chance zu entkommen.

Ihnen als medizinische Fachkräfte kommt dabei eine entscheidende Rolle zu. Diese Kampagne soll Ihnen helfen zu erkennen, ob Ihre Patientin oder Ihr Patient ein Opfer von Menschenhandel ist.

\section{Jeder Fall ist anders}

Es ist schwierig, Menschenhandelsopfer zu erkennen. Die Anzeichen von Not und Hilflosigkeit sind subtil und nicht auf den ersten Blick ersichtlich. Das typische

\section{Es ist schwierig, Menschenhandelsopfer zu erkennen.}

Opfer gibt es nicht; jeder Fall ist anders. Man muss die physischen und psychischen Anzeichen und schliesslich auch die Umstände deuten können, die darauf schliessen lassen, dass man ein Opfer von Menschenhandel vor sich hat. Eine Frau wird zum Beispiel von einem Mann begleitet, der durch sein aggressives Verhalten auffällt und darauf besteht, bei der Untersuchung dabei zu sein. Die Patientin spricht nur in

\section{Was kann ich konkret tun?}

Wenn Sie vermuten, dass Sie es mit einem Opfer von Menschenhandel zu tun haben, zögern Sie nicht zu handeln. Menschenhandelsopfer sind verletzlich und psychisch labil. Oft misstrauen sie den Behörden. Sie fürchten Repressalien, zum Beispiel wenn sie mit der Polizei sprechen. Eine erste einfache Hilfsmassnahme kann darin bestehen, sie mit einer Opferhilfeeinrichtung in Kontakt zu bringen. Zu diesem Zweck ist eine Pocket Card mit einer Notfalladresse kreiert worden. Geben Sie diese dem Opfer. So kann es zu einem späteren Zeitpunkt entscheiden, ob es Hilfe in Anspruch nehmen möchte oder nicht.

Im schlimmsten Fall gibt Ihnen die Patientin oder der Patient gar keine Auskunft. Oder aber die angegebene Ursache der körperlichen Verletzungen vermag diese nicht wirklich zu erklären. Sie können Ihrem Gegenüber klarmachen, dass es einen Ausweg gibt. Überreichen Sie die Pocket Card, und sagen Sie beispielsweise: "Mir ist klar, dass Sie im Moment nichts tun können. Aber nehmen Sie diese Karte. Vielleicht nützen Ihnen die Informationen später einmal.»

Im besten aller Fälle ist die Patientin oder der Patient offen für das, was Sie sagen. Erklären Sie Ihrem Gegenüber, dass es Rechte hat und dass Sie den Kontakt zu einer Opferhilfeeinrichtung oder zu den kantonalen Polizeibehörden herstellen können. Dazu brauchen Sie allerdings das Einverständnis der Patientin oder des Patienten.

einer fremden Sprache, blickt ängstlich um sich oder vermeidet den direkten Blickkontakt. Die Erklärungen, woher die Verletzungen rühren, sind unzureichend.

Ein in Spitälern und Arztpraxen verteilter Informationsflyer listet diese und andere mögliche Anzeichen auf. Die medizinischen Fachkräfte sollen in der Lage sein, Menschenhandelsopfer einfacher zu erkennen, um sie zu beschützen und ihnen helfen zu können. $\mathrm{Zu}$ diesem Flyer gibt es auch einen Kurzfilm, der die Schwierigkeiten aufzeigt, Opfern von Menschenhandel zu helfen.

\section{Gewinnen Sie Zeit und das Vertrauen der Patientin oder des Patienten}

Medizinisches Fachpersonal kann mit solchen Situationen zwar durchaus umgehen. Allerdings ist es nicht immer leicht, im Alltag in der Notaufnahme stets angemessen zu reagieren. Dazu braucht es Erfahrung und das Vermögen zu erkennen, was nicht ausgesprochen wird. Während der Untersuchung soll das medizinische Fachpersonal nicht zögern, viele Fragen zu stellen und möglichst viel in Erfahrung zu bringen. Spricht die Patientin eine fremde Sprache, kann ein Dolmetscherdienst hinzugezogen werden. Wichtig ist, Zeit und das Vertrauen des mutmasslichen Opfers zu gewinnen, um es aufklären zu können (siehe Kasten). 


\section{Was können Opfer erwarten?}

In der Schweiz hat ein Opfer von Menschenhandel Rechte, die es geltend machen kann. Es hat ungeachtet seiner Nationalität oder seines Aufenthaltsstatus Anspruch auf Unterstützung und Hilfe. Es hat ein Recht

\section{In der Schweiz hat ein Opfer von Menschen- handel Rechte, die es geltend machen kann.}

darauf, beschützt zu werden, indem ihm bei der Suche nach einer Unterkunft geholfen wird und es medizinische und juristische Unterstützung erhält. Ist der Kontakt mit einer Opferhilfeeinrichtung erst einmal hergestellt, wird alles daran gesetzt, um das Opfer zu beschützen und dafür zu sorgen, dass es wieder Stabilität in sein Leben bringen kann. Dies gilt umso mehr, wenn das Opfer sich illegal in der Schweiz aufhält. Ist ein Opfer dazu bereit, kann es seinen Peiniger anzeigen und bei der Polizei eine Aussage machen. Während der Ermittlungen kann dem Opfer eine Aufenthaltsbewilligung erteilt werden, und während wie auch nach dem Strafverfahren geniesst das Opfer Zeugenschutz.

\section{Menschenhandel betrifft auch die Schweiz}

2017 wurden in der Schweiz 149 Opfer identifiziert. Menschenhandel gibt es in unterschiedlichen Formen und in verschiedenen Sektoren. Zwangsprostitution ist die am weitesten verbreitete Form von Menschenhandel. Die Opfer sind junge Frauen, oft aus Südosteuropa. Auch zur Ausbeutung der Arbeitskraft wird mit Menschen gehandelt. In der 2016 von der Universität Neuenburg veröffentlichten Studie "Arbeitsausbeutung im Kontext von Menschenhandel» gehen die Experten von einer hohen Dunkelziffer aus. Sie vermuten, dass Ausbeutung in verschiedenen Branchen vorkommt: im Baugewerbe, in der Hotellerie, im Gastgewerbe sowie in der Haus- und Landwirtschaft. Sowohl Frauen als auch Männer und Minderjährige können Opfer von Menschenhandel werden. Es handelt sich um verletzliche Menschen, die in ihrem Heimatland in Armut leben. In der Hoffnung auf ein besseres Leben in der Schweiz sind sie bereit, das Schlimmste zu ertragen. Indem Sie ein Opfer aufklären und seine Notlage erkennen, können Sie bereits helfen, sein Leben zu verändern.

Bildnachweis

(c) Michael Philipp, luxs

\section{Menschenhandel - Was ist das?}

Menschenhandel besteht im Anwerben, Verbringen, Vermitteln, Beherbergen oder Annehmen von Menschen zum Zweck der Ausbeutung. Die Opfer werden mit illegalen Mitteln genötigt, beispielsweise indem ihre Hilflosigkeit ausgenutzt und physische oder psychische Gewalt angewendet wird. Die Ausbeutung kann in der sexuellen Ausbeutung oder der Ausbeutung der Arbeitskraft bestehen. Die Opfer werden wie Ware behandelt und verlieren jegliches Selbstbestimmungsrecht. 